\title{
The spatial-temporal distribution and aetiological characteristics of hand, foot, and mouth disease before and after the EV-A71 vaccination and COVID- 19 pandemic in Kunming, China, 2017-2020
}

\section{Meifen Wang}

Kunming Children's Hospital

\section{Tao Chen}

The Affiliated Hospital of Yunnan Normal University

Junchao Peng

Kunming Children's Hospital

Yunjiao Luo

Kunming Children's Hospital

Lijiang Du

Kunming Children's Hospital

Zhiying Lu

Kunming Children's Hospital

Jianzhu He

Kunming Children's Hospital

Chunli Liu

Kunming Children's Hospital

Quan Gan

Kunming Children's Hospital

\section{Wei Ma}

Kunming Children's Hospital

\section{Zhikuan Cun}

Kunming Children's Hospital

Qiongmei Zheng

Kunming Children's Hospital

\section{Weiying Chen}

Kunming Children's Hospital

\section{Yonglin Chen}

First Affiliated Hospital of Kunming Medical University

Mei Han

Weather Modification Center of Yunnan Province 
guojun liu

Weifang Center for Disease Control and Prevention

Jiwei Li (D360623155@qq.com )

Kunming Children's Hospital

\section{Research Article}

Keywords: Hand-foot-and-mouth disease, Enterovirus A71 vaccine, COVID-19 pandemic, Spatial-temporal distribution, Epidemiological characteristics

Posted Date: January 20th, 2022

DOI: https://doi.org/10.21203/rs.3.rs-1218319/v1

License: (c) (i) This work is licensed under a Creative Commons Attribution 4.0 International License.

Read Full License 


\section{Abstract \\ Background}

After the vaccination enterovirus 71 (EV-A71) vaccine and Corona Virus Disease 2019 (COVID-19) outbreak, the prevalence of Hand, foot, and mouth disease (HFMD) remained high and the spatialtemporal distribution of enteroviruses changed. Therefore, it is essential to define the temporal features, spatial distributions, epidemiological and aetiological characteristics of HFMD of Kunming.

\section{Methods}

Between 2017 and 2020, a total of 36540 HFMD children cases diagnosed with HFMD in Kunming, including 32754 enteroviruses positive clinical samples. Demographic, geographical, epidemiological and aetiological data of the cases were acquired and analyzed.

\section{Results}

Other enteroviruses replaced EV-A71 and the incidence of EV-A71 has decreased dramatically, while, coxsackievirus A6 (Cox A6) and coxsackievirus A16 (Cox A16) with significant outbreaks in 2018 and 2019 , respectively. The major and junior peaks all extended for 2-4 months than before that vaccination EV-A71 vaccine. After the COVID-19 outbreak, Cox A6 as the predominant serotype pathogens, and only single peaks appeared in 2019 and 2020. Although the high incidence of HFMD areas were Guandu, Chenggong and Xishan, the annual incidence of different enterovirus serotype appeard in different regions. In 2017, other enteroviruses prevailed in Shilin; In 2018, Cox A16 and Cox A6 prevailed in Luquan and Shilin, respectively; In 2019, Cox A16 prevailed in Jinning; In 2020, Cox A6 and coxsackievirus A10 (Cox A10) prevailed in Luquan and Shilin, respectively. Meanwhile, the epidemic cycle of Cox A6 and Cox A16 was only 1 year, and Cox A10 and other enteroviruses were potential risk pathogens.

\section{Conclusions}

The spatial and temporal distribution of HFMD varies at different scales, and the incidence of different pathogens associated HFMD has obvious regional differences and seasonal trends. Therefore, the research of multivalent combined vaccines are urgently needed, while, proper preventive and protective measures could effectively control the incidence of HFMD-like diseases.

\section{Introduction}

Since 1997, HFMD has been primarily prevalent in the Asia-Pacific region, including China, Japan, Singapore, Malaysia, Vietnam, South Korea, Thailand, Cuba, India and Cambodia. In mainland China, with an incidence of $37.01 / 100,000-205.06 / 100,000^{[1,2]}$, a total of 23.5 million HFMD cases have been 
reported to the Chinese Center for Disease Control and Prevention (CDC) during 2000-2018 ${ }^{[3,4]}$, and showed an increasing trend continuously in recent years. Previous studies have focused on the eastern coastal, densely populated and economically developed cities(including Shandong, Anhui, Jiangxi, Jiangsu, Zhejiang, Fujian, Guangdong province, Beijing, Shanghai and Shenzhen municipality), and indicated that the epidemiological characteristics of EV-A71 and Cox A16, which were considered the most common aetiological agents of HFMD in the past two decades ${ }^{[5]}$. But less was known about what caused the different characteristics of the descriptive epidemiology of HFMD between different areas ${ }^{[6-}$ 10].

In order to control the epidemic of HFMD, the inactivated monovalent EV-A71 vaccine was wildly vaccinated throughout China since 2016, which was highly effective against EV71-associated HFMD ${ }^{[11}$, ${ }^{12]}$, but the prevalence of HFMD induced by other serotypes remain high ${ }^{[13,14]}$. Meanwhile, due to the COVID-19 pandemic, the public areas were closed and quarantine of infected patients were imposed since December 2019. We have come to realize the regional surveillance of epidemics and no physical contact with infected patients, which played significant role in reducing HFMD-like diseases cases in 2020.

As a medium-sized inland developing city in Southwest China with high altitude and complex terrain, Kunming urgently needs research on the temporal features, spatial distributions, epidemiological and aetiological characteristics of HFMD, with an in-depth analysis before and after the EV-A71 vaccination and COVID-19 pandemic.

\section{Materials And Methods}

\section{Study area}

Kunming is the first largest city of Yunnan Province in Southwest China. Within an area of approximately 21473 square kilometres, the city has complex terrain consisting of mountains, hills, plains, basins and plateaus, and subtropical monsoon continental climate. It administers 7 districts, 1 county-level cities, 3 counties and 3 autonomous counties, and has a population of 8.46 million people now, including $1,267,713$ people aged $0-14$, accounting for $14.98 \%$.

\section{Data collection}

We collected surveillance data on the prevalence of HFMD in Kunming Children's Hospital from 2017 to 2020, including gender, age, population classification, address, type of diagnosis and laboratory test results (confirmed by real-time RT-q PCR in the clinical laboratory of the hospital), of 36540 HFMD cases.

\section{Clinical definition}

According to the guidelines of the National Health Commission of the People's Republic of China, all 32754 enteroviruses positive clinical samples contained the following 4 clinical manifestations: HFMD, 
Severe HFMD, Herpangina, and fever. Severe HFMD patients were at least one of the following: cardiopulmonary collapse, pulmonary hemorrhage, pulmonary edema, encephalitis, aseptic meningitis, acute flaccid paralysis, yocarditis, or death. Laboratory test results were divided into 6 categories: EV-A71 positive, Cox A16 positive, Cox A6 positive, Cox A10 positive and other enteroviruses positive and enterovirus negative.

\section{Statistical analysis}

Descriptive epidemiological methods were used to analyse the demographic (including age, sex and population classification), aetiological (EV-A71, Cox A16, Cox A6, Cox A10 and other enteroviruses), temporal and spatial characteristics of HFMD by month.

SPSS 22.0 statistical software was used for statistical analysis. The basic situation was analyzed by descriptive analysis, and the counting data was analyzed by chi-square test. Analyze the inspection and check the level $a=0.05$. The geographic distribution map of HFMD cases was drawn by Maplnfo 10.0 spatial distribution software.

\section{Ethics statement}

All the study procedures were reviewed and approved by the Ethics Committee of Kunming Children's Hospital. Written consent was obtained from each participant patient's parents or guardians in the study.

\section{Results}

\section{Demographic characteristics}

Total 32754 positive cases of HFMD were monitored from 2017 to 2020 in Kunming, the annual incidence rates were $71.58 / 100,000$ at minimum and $163.24 / 100,000$ at maximum, and the average incidence rate was 118.97/100,000 (Table 2). HFMD patients under 5 years predominated and accounting for $93.30 \%, 52.17 \%$ of HFMD patients were aged $0-3$ years, and $41.13 \%$ were aged $3-5$ years. The male-to-female ratio of HFMD cases was 1.46:1 (ranging from 1.43:1 to 1.49:1) and showed a steady trend. The first highest proportion of classified HFMD cases was observed in scattered children, accounting for $55.77 \%$, the second highest proportion was nursery children, accounting for $39.97 \%$, and the proportions of school-children was small, accounting for $4.26 \%$ (Table 1 ).

\section{Enterovirus serotype distribution}

The numbers of serotypes pathogens of laboratory-confirmed cases fluctuated during 2017-2020, and we observed that there are two important time inflection points of change of enterovirus serotype in 2017 and 2020, respectively. The annual number of EV-A71 was $10.4 \%, 3.8 \%, 1.3 \%$, and only two cases, which decreased continuously. The number of other enteroviruses and Cox A16 first trended upward and then decreased. Other enteroviruses increased significantly and accounted for $76.2 \%$ in 2017 , but decreased year by year, $28.8 \%$ in 2018, $16.3 \%$ in 2019 , and $17.9 \%$ in 2020 . While Cox A16 increased gradually and 
accounted for $30.0 \%, 47.2 \%$ in 2018 and 2019 respectively, but decreased dramatically to $4.1 \%$ in 2020 . The number of Cox A6 and Cox A10 trended upward continuously, particularly, Cox A6 increased significantly and accounted for 33.3\%, 33.7\% in 2018 and 2019 respectively, and suddenly rose to $70.8 \%$ in 2020. The annual number of Cox A10 was maintained at a low level from 2017 to $2019,0.2 \%, 4.0 \%$, $1.6 \%$, respectively, but increased to $7.1 \%$ in 2020 (Table 1 and Figure 1 ).

\section{Temporal characteristics}

The annual incidence of HFMD in Kunming initially increased and presented a seasonal distribution with semi-annual or annual peaks. Two peaks were observed in 2017 and 2018, a major one in April to June and the other in October to November, and the peaks value in 2018 was much higher than that in 2017. Single peak appeared in 2019 and 2020, although the peaks value were close, they showed obvious differences. The peak in 2019 followed by a platform shape until next February; In 2020, the only one peak from July to next February (Figure 2).

\section{Spatial characteristics}

During 2017-2020, 7 districts, 1 county-level cities, 3 counties and 3 autonomous counties of Kunming reported the occurrence of HFMD. The spatial distribution of HFMD incidence was heterogeneous at the county level, ranging from 1.52/100,000 (Shilin) to 384.51/100,000 (Guandu). The counties with the highest incidence of HFMD were Guandu, Chenggong, Xishan, Songming, Wuhua and Panlong. In some regions, such as Luquan, the incidence increased gradually from 14.97/100,000 in 2017 to $78.17 / 100,000$ in 2018 and $81.02 / 100,000$ in 2020 . Overall, the relatively high-incidence areas were the core area of Kunming with concentrated population and prosperous economy (Table 2 and Figure 3).

\section{Spatio-temporal clusters}

During 2017-2020, the periodic peaks of the HFMD presented the timing of the spatio-temporal clusters, and different pathogens showed the different spatial-temporal distribution (Figure 4). In 2017, the areas with high incidence of other enteroviruses were Shilin (100\%), Yiliang (94.7\%), Anning (89.3\%), Chenggong (85.1\%) and Luquan (79.0\%). In 2018, the areas with high incidence of Cox A16 were Luquan (43.5\%), Xundian (39.3\%), Fumin (37.6\%) and Guandu (33.2\%); the areas with high incidence of Cox A6 were Shilin (60.0\%), Dongchuan (49.5\%), Jinning (45.8\%), Chenggong (37.8\%) and Wuhua (36.5\%). In 2019 , the areas with high incidence of Cox A16 were Jinning (59.9\%), Yiliang (55.8\%), Chenggong (49.5\%), Panlong (48.4\%) and Anning (48.2\%). In 2020, the areas with high incidence of Cox A6 were Luquan (77.4\%), Dongchuan (75.7\%), Anning (74.5\%), Fumin (74.1\%) and Xundian (73.4\%); the areas with high incidence of Cox A10 were Shilin (50.0\%), Songming (19.1\%) and Yiliang (17.1\%).

\section{Discussion}

According to World Health Organization (WHO) reports, China is one of the countries with high incidence of HFMD ${ }^{[15]}$. Since 2008 the outbreak, HFMD has been listed as the type $C$ statutorily notifiable infectious 
diseases $^{[16]}$ and under constant surveillance. The east coastal cities of China have carried out earlier researches and summarized some regional epidemiological characteristics of HFMD, including the prevalence in children under five years old ${ }^{[17]}$, with a higher incidence in male in local residents, one peak in a year ${ }^{[18]}$, and EV-A71 was the most common causative pathogen ${ }^{[1,7,19]}$.

In recent years, central and western cities of China, like Sichuan and Xinjiang, also have gradually conducted research on HFMD and found obviously regional epidemiological characteristics, such as two peak epidemic seasons in a year ${ }^{[1]}$, and EV-A71 and Cox A16 were considered as the common aetiological agents in local areas ${ }^{[7,21]}$. Kunming, as a southwest inland city of China, also has made constant surveillance with HFMD for over a decade. A few reports showed the research results of the local incidence of HFMD before 2017, and found that children aged 1-3 and scattered residence were at the highest risk, the ratio of male to female was 1.5:1, a major peak during April to June followed by a autumn peak during October to November, and EV-A71, Cox A16, and other enteroviruses were the most common causative pathogens ${ }^{[22]}$. These results reflected some regional epidemiological and etiological characteristics of HFMD in Kunming before the vaccination of EV-A71 vaccine, and played a certain role in the prevention and control.

Since 2016, the inactivated monovalent EV-A71 vaccine has been inoculated nationwide in China, which significantly reduces the EV-A71-associated HFMD over $90 \%{ }^{[23]}$. However, due to no cross-protection from the vaccine and the high rate of gene mutation and recombination in other serotypes ${ }^{[24]}$, the prevalence of HFMD remain high, such as Cox A16 replaced EV-A71 in Guangzhou, Shenzhen and Xinjiang; Cox A6 as the dominant serotypes pathogens prevailed in Xiangyang and Sichuan ${ }^{[4,14]}$. Kunming, from 2017 to 2020 , about 600,000 doses of inactivated monovalent EV-A71 vaccines have been vaccinated by the local CDC, and the incidence of EV-A71-associated HFMD has decreased dramatically, $10.4 \%$ in 2017 and only $1.3 \%$ in 2019 . In 2017 , other enteroviruses replaced EV-A71 as the predominant serotype pathogen and accounted for 76.2\%. However, Cox A6 and Cox A16 showed an increasing trend from 2017 to 2019 , with significant outbreaks in 2018 (33.3\%) and 2019 (47.2\%), respectively. Meanwhile, the peak time was longer than that before vaccination EV-A71 vaccine, the major peak extended for two months from April to August, and the junior peak extended for four months from September to next February. This phenomenon mainly due to the vaccination EV-A71 vaccine impart selective pressure for these other serotypes pathogens to emerge at high proportions ${ }^{[25]}$, and the different enterovirus genotypes with dissimilar activity and transmission characteristics, under the influences of complex social and climatic factors ${ }^{[7,26]}$, which can cause differing scales and peak patterns ${ }^{[27]}$.

In addition, after the COVID-19 outbreak, we found that the incidence of Cox A6 and Cox A10 has increased significantly, and Cox A6 replaced Cox A16 as the predominant serotype pathogens and accounted for $70.8 \%$. From the end of 2019 , the junior peak disappeared and presented a platform shape until next February. In 2020, it only presented a single peak, and continued from July to next February. We speculate that the main reasons are as follows. First, the effective personal hygiene propaganda during the epidemic period, such as reducing physical contact, wearing masks and washing hands, which 
effectively reduced the transmission route of HFMD. Second, public places were closed and children with HFMD were completely isolated at home. Third, the surveillance of population flow has obviously affected the epidemic and outbreak cycle of different pathogens.

In order to further understand the evolutionary dynamics of HFMD enterovirus and enhance the vaccination strategies in Kunming, we introduce the spatial and temporal description of the epidemiological and aetiological characteristics. The spatial-temporal scanning includes geographic information, corrects the non-uniform population density in different places, makes up for the deficiency of the simple epidemiological morbidity comparison, objectively and comprehensively evaluates the abnormal increase and the incidence aggregation area in both time and space dimensions ${ }^{[28]}$.

From 2017 to 2020, by using spatial-temporal scanning, we analyzed the number of cases of HFMD mainly concentrated in Guandu, Xishan, Wuhua, Panlong and Chenggong, and the high incidence of HFMD areas were Guandu, Chenggong and Xishan. Both the number and incidence of HFMD presented outbreak in 2018 and 2019, and continued to increase in Chenggong. Spatial aggregation means that the risk of HFMD is significantly higher in some areas than in others, probably because of more residential and high population density, which can lead to clustered infection. In addition, it is interesting to note that these areas are mainly concentrated near the Panlong river and Dianchi lake, which indicates that the prevalence of HFMD may be associated with the humidity, pollution or other factors related to the lake. Moreover, different pathogens have different spatial and temporal distribution, and which also occurs in the same pathogen. In 2017, other enteroviruses as the predominant serotype pathogens, the areas with high proportion were Shilin, Yiliang and Anning. In 2018, Cox A16 was mainly prevalent in Luquan, Xundian and Fumin; Cox A6 was mainly prevalent in Shilin, Dongchuan and Jinning. In 2019, Cox A16 as the predominant serotype pathogens, the areas with high proportion were Jinning, Yiliang and Chenggong. In 2020, Cox A6 as the predominant serotype pathogens, the areas with high proportion were Luquan, Dongchuan and Anning; Cox A10 was mainly prevalent in Shilin, Songming and Yiliang. Although the annual total incidence of HFMD is directly related to population density, the annual incidence of different enterovirus serotype appears in different regions, which will bring great challenges to the epidemic prevention work of HFMD. It was reported that the epidemics of EV-A71 or Cox A16 circulates in a cyclical pattern of every 2-3 years ${ }^{[29]}$. Throughout the Kunming after vaccination EV-A71 vaccines, we have noticed that other enteroviruses has decreased significantly since 2018, but by 2019 , it has remained stable at 15\% to 20\%; Cox A16 gradually increased from 2018 and broke out in 2019; Cox A6 broke out regionally in 2018, and gradually increased in 2019; Cox A10 increased since 2018. After the COVID-19 pandemic, Cox A6 broke out throughout the Kunming, Cox A10 broke out regionally, and other enteroviruses remained stable at $20 \%$ and surpassed Cox A6. We found that the epidemics and outbreaks of Cox A6 and Cox A16 associated HFMD appeared alternately, and the cycle was only 1 year; Cox A10 and other enteroviruses, although the incidence was not high, they were possible associated potential risk pathogens for outbreaks of HFMD in the next cycle period.

This study has several limitations should be acknowledged. First, HFMD are asymptomatic and selflimiting, thus part cases of HFMD may be ignored through the passive surveillance system, and the 
actual cases may have been underestimated. Second, we did not test the enterovirus serotypes beyond EV-A71, Cox A16, Cox A6, Cox A10 and other serotypes, because that which had not been dominant in the past based on other studies. Third, we analyzed the association between the spatial and temporal distribution of infectious diseases, but the spread of disease is influenced by a variety of factors, such as meteorological, socioeconomic, health resource and traffic variables. However, due to limited access to data, these factors were not considered in this study.

\section{Conclusions}

This study confirms that the spatial and temporal distribution of HFMD varies at different scales, and the incidence of different pathogens associated HFMD has obvious regional differences and seasonal trends. Therefore, the research and development of multivalent combined vaccines including EV-A71, Cox A16, Cox A6 and Cox A10 are urgently needed, which could cover over $90 \%$ of pathogens associated HFMD in the southwest region. In addition, due to the COVID-19 pandemic, the number of infected patients was significantly low for 2020. So, taking proper preventive and protective measures could effectively control the incidence of HFMD-like diseases in Kunming and even nationwide.

\section{Declarations}

\section{Statement}

All methods were carried out in accordance with relevant guidelines and regulations.

\section{Acknowledgments}

The manuscript is an original work and has not been published or is under consideration for publication in another journal. The study complies with current ethical consideration. The authors confirmed that all the listed authors have participated actively in the research, and have seen and approved the submitted manuscript.

\section{Conflict of Interest Statement}

We declare that we have no conflict of interest.

\section{Funding}

This work was supported by the Kunming "Spring City Plan" high-level talent training project, Kunming municipal government "spring city famous doctor" special project [grant number 2020-23]; Kunming Science and technology planning project [grant number 2019-1-S-25318000001241]; Kunming health and Family Planning Commission Project [grant number 2019-SW-33]; Yunnan Province's reserve medical talents project [grant number H-2019002]; Kunming Medical University Applied Basic Research Joint Special Project [grant number 202001AY070001-170]. 


\section{References}

1. Puenpa, J. et al. Hand, Foot, and Mouth Disease Caused by Coxsackievirus A6, Thailand, 2012.[J]. Emerg Infect Dis, 2013, 19(4): 641-3.

2. Saki T, Liao Q, Van B, et al. Hand, Foot, and Mouth Disease in China: Modeling Epidemic Dynamics of Enterovirus Serotypes and Implications for Vaccination.[J]. Plos Medicine, 2016, 13(2):e1001958.

3. Sumi A, Toyoda S, Kanou K et al. Association between meteorological factors and reported cases of hand, foot, and mouth disease from 2000 to 2015 in Japan.[J]. Epidemiol Infect, 2017, 145(14):2896-911.

4. Yang B, Liu F, Liao Q et al. Epidemiology of hand, foot and mouth disease in China, 2008 to 2015 prior to the introduction of EV-A71 vaccine.[J]. Euro Surveill, 2017, 22(50):38-47.

5. Qi H, Li Y, Zhang J, et al. Quantifying the risk of hand, foot, and mouth disease (HFMD) attributable to meteorological factors in East China: A time series modelling study.[J]. Science of The Total Environment, 2020, 728:138548.

6. Liang YK, Li N, Yang JZ, Deng B, Xie RH, Shu S, et al. Epidemiologic characteristics of hand-footmouth disease in Guiyang between 2008 and 2010.[J]. Zhongguo Dang Dai Er Ke Za Zhi, 2012, 14(3):195-7.

7. Xing W, Liao Q, Viboud C, Zhang J, Sun J, Wu JT, et al. Hand, foot, and mouth disease in China, 200812: an epidemiological study.[J]. The Lancet. Infectious Diseases, 2014, 14(4):308-18.

8. Sun LM, Wu SL, Tan XH, Li H, Yang F, Zeng HR, et al. Epidemiological characteristics of Coxsackie virus A16 caused hand foot and mouth disease cases in Guangdong province, 2012-2016.[J]. Zhonghua Liu Xing Bing Xue Za Zhi, 2018, 39(3):342-346.

9. Li Y, Bao H, Zhang X, Zhai M, Bao X, Wang D, et al. Epidemiological and genetic analysis concerning the non-enterovirus 71 and non-coxsackievirus A16 causative agents related to hand, foot and mouth disease in Anyang city, Henan Province, China, from 2011 to 2015.[J]. Journal of Medical Virology, 2017,89(10):1749-58.

10. Chen Q, Xing XS, Wu Y, Liao QH, Liu GP, Jiang XQ, et al. Hand, foot and mouth disease in Hubei province, 2009-2015: an epidemiological and etiological study.[J]. Zhonghua Liu Xing Bing Xue Za Zhi, 2017,38(4):441-5.

11. Li R, Liu L, Mo Z, et al. An inactivated enterovirus 71 vaccine in healthy children.[J]. N Engl J Med, 2014, 370(9):829-37.

12. Zhu F, Xu W, Xia J, et al. Efficacy, safety, and immunogenicity of an enterovirus 71 vaccine in China. [J]. N Engl J Med, 2014, 370(9):818-28.

13. Zhou Y, Li JX, Jin PF, et al. Enterovirus 71: a whole virion inactivated enterovirus 71 vaccine.[J]. Expert Rev Vac, 2016, 15(7):803-13.

14. Zhang J. Trend of epidemics and variation of pathogens of hand, foot and mouth disease in China: a dynamic series analysis, 2008-2017.[J]. Chinese Journal of Endemiology, 2019, 40(2):147-154. 
15. Owino CO, Chu J. Recent advances on the role of host factors during non-poliovirus enteroviral infections.[J]. Journal of Biomedical Science, 2019, 26(1):1-17.

16. Jian $\mathrm{C}, \mathrm{Wu} \mathrm{J}, \mathrm{Xu} Z$, et al. Associations between extreme precipitation and childhood hand, foot and mouth disease in urban and rural areas in Hefei, China.[J]. Science of the Total Environment, 2014, 497-498(nov.1):484-490.

17. Yang S, Wu J, Ding C et al. Epidemiological features of and changes in incidence of infectious diseases in China in the first decade after the SARS outbreak: an observational trend study.[J]. Lancet Infect Dis, 2017, 17(7):716-25.

18. Wang J, Teng Z, Cui X et al. Epidemiological and serological surveillance of hand-foot-and-mouth disease in Shanghai, China, 2012-2016.[J]. Emerg Microbes Infect, 2018,7(1):8.

19. Mong, How, Ooi, et al. Clinical features, diagnosis, and management of enterovirus 71.[J]. Lancet Neurology, 2010, 9(11):1097-1105.

20. Gopalkrishna V, Patil PR, Patil GP, et al. Circulation of multiple enterovirus serotypes causing hand, foot and mouth disease in India.[J]. J Med Microbiol, 2012, 61(3):420-5.

21. Weng Yuwei,Chen Wei,Huang Meng et al. Epidemiology and etiology of hand, foot, and mouth disease in Fujian province, 2008-2014.[J].Arch Virol, 2017, 162(2): 535-542.

22. Jiang Hongchao, Zhang Zhen, Rao Qing et al. The epidemiological characteristics of enterovirus infection before and after the use of enterovirus 71 inactivated vaccine in Kunming, China.[J].Emerg Microbes Infect, 2021, 10(1): 619-628.

23. Lin JY, Kung YA, Shih SR. Antivirals and vaccines for Enterovirus A71.[J].J Biomed Sci, 2019, 26(1): 65.

24. Nicholson Erin, Piedra Pedro A. Local Versus Global Enterovirus (EV) Surveillance: A Discussion for the Need for Active Surveillance to Guide EV-A71 Vaccines.[J].J Infect Dis, 2017, 216(11): 13371339.

25. Meng X D, Tong Y, Wei Z N, et al. Epidemical and etiological study on hand, foot and mouth disease following EV-A71 vaccination in Xiangyang, China[J]. Scientific Reports, 2020,10(1):20909.

26. Chen M, He S, Yan Q et al. Severe hand, foot and mouth disease associated with Coxsackievirus A10 infections in Xiamen, China in 2015.[J].Clin Virol, 2017, 93:20-24.

27. Peng Di,Ma Yue,Liu Yaqiong et al. Epidemiological and aetiological characteristics of hand, foot, and mouth disease in Sichuan Province, China, 2011-2017.[J]. Sci Rep, 2020, 10(1): 6117.

28. Wang Yanhao,Zhao Han,Ou Rong et al. Epidemiological and clinical characteristics of severe handfoot-and-mouth disease (HFMD) among children: a 6-year population-based study.[J]. BMC Public Health, 2020, 20(1): 801.

29. Mao Q, Wang Y, Shao J, et al. The compatibility of inactivated-enterovirus 71 vaccination with coxsackievirus A16 and poliovirus immunizations in humans and animals.[J]. Hum Vaccin Immunother, 2015,11(11):2723-2733. 


\section{Tables}

Table 1. The epidemiological description of HFMD in Kunming, 2017-2020. 


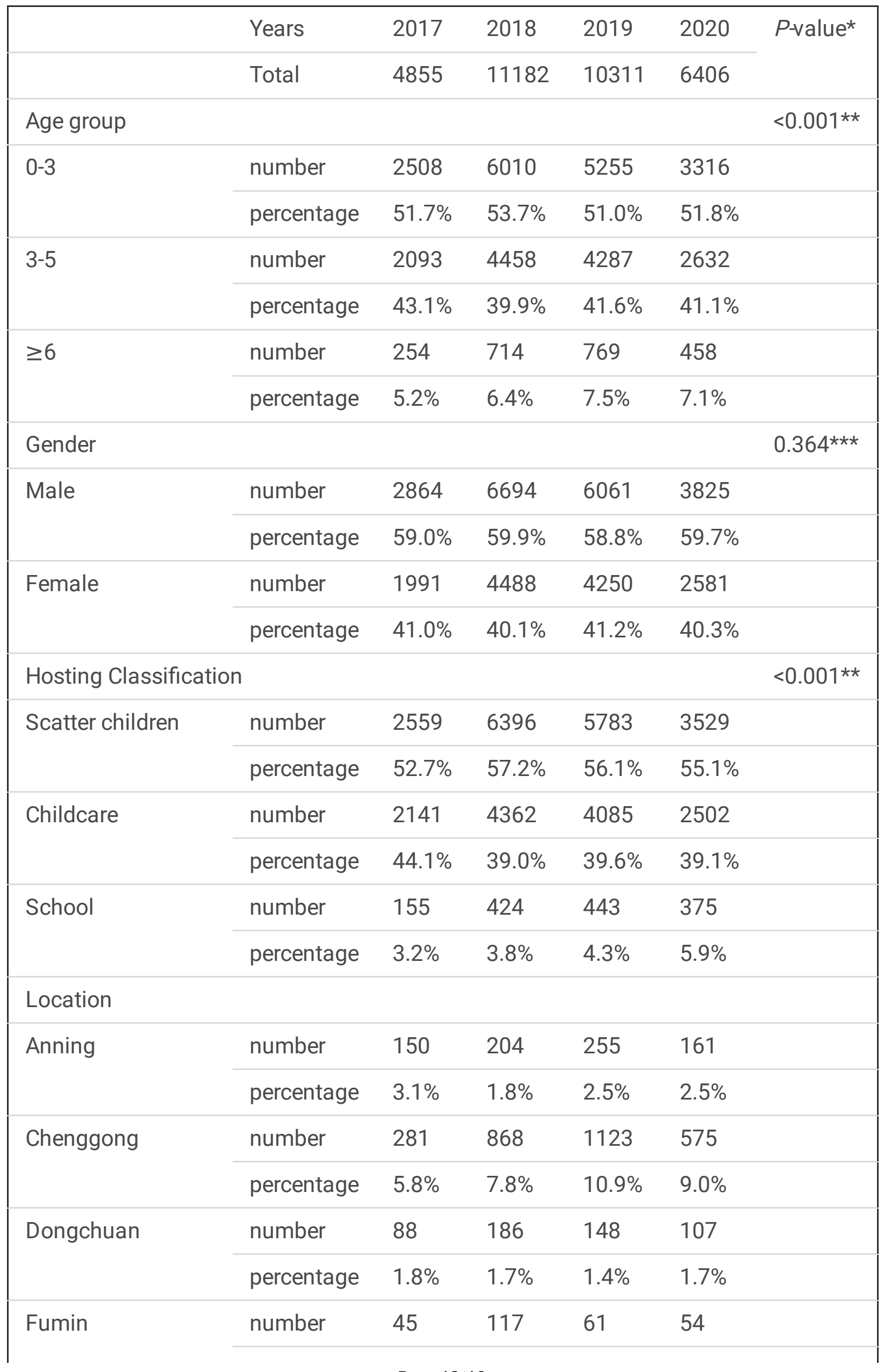




\begin{tabular}{|c|c|c|c|c|c|}
\hline & percentage & $0.9 \%$ & $1.0 \%$ & $0.6 \%$ & $0.8 \%$ \\
\hline \multirow[t]{2}{*}{ Guandu } & number & 1584 & 3514 & 3250 & 1934 \\
\hline & percentage & $32.6 \%$ & $31.4 \%$ & $31.5 \%$ & $30.2 \%$ \\
\hline \multirow[t]{2}{*}{ Jinning } & number & 118 & 277 & 217 & 116 \\
\hline & percentage & $2.4 \%$ & $2.5 \%$ & $2.1 \%$ & $1.8 \%$ \\
\hline \multirow[t]{2}{*}{ Luquan } & number & 62 & 324 & 188 & 336 \\
\hline & percentage & $1.3 \%$ & $2.9 \%$ & $1.8 \%$ & $5.2 \%$ \\
\hline \multirow[t]{2}{*}{ Panlong } & number & 565 & 1255 & 1127 & 659 \\
\hline & percentage & $11.6 \%$ & $11.2 \%$ & $10.9 \%$ & $10.3 \%$ \\
\hline \multirow[t]{2}{*}{ Shilin } & number & 4 & 30 & 24 & 14 \\
\hline & percentage & $0.1 \%$ & $0.3 \%$ & $0.2 \%$ & $0.2 \%$ \\
\hline \multirow[t]{2}{*}{ Songming } & number & 253 & 573 & 559 & 262 \\
\hline & percentage & $5.2 \%$ & $5.1 \%$ & $5.4 \%$ & $4.1 \%$ \\
\hline \multirow[t]{2}{*}{ Wuhua } & number & 661 & 1360 & 1109 & 907 \\
\hline & percentage & $13.6 \%$ & $12.2 \%$ & $10.8 \%$ & $14.2 \%$ \\
\hline \multirow[t]{2}{*}{ Xishan } & number & 859 & 1869 & 1735 & 1051 \\
\hline & percentage & $17.7 \%$ & $16.7 \%$ & $16.8 \%$ & $16.4 \%$ \\
\hline \multirow[t]{2}{*}{ Xundian } & number & 91 & 336 & 249 & 154 \\
\hline & percentage & $1.9 \%$ & $3.0 \%$ & $2.4 \%$ & $2.4 \%$ \\
\hline \multirow[t]{2}{*}{ Yiliang } & number & 94 & 269 & 267 & 76 \\
\hline & percentage & $1.9 \%$ & $2.4 \%$ & $2.6 \%$ & $1.2 \%$ \\
\hline \multicolumn{6}{|c|}{ Identification of enterovirus } \\
\hline \multirow[t]{2}{*}{ EV-A71 } & number & 504 & 430 & 130 & 2 \\
\hline & percentage & $10.4 \%$ & $3.8 \%$ & $1.3 \%$ & $0.0 \%$ \\
\hline \multirow[t]{2}{*}{ Cox A16 } & number & 629 & 3359 & 4866 & 263 \\
\hline & percentage & $13.0 \%$ & $30.0 \%$ & $47.2 \%$ & $4.1 \%$ \\
\hline \multirow[t]{2}{*}{ Cox A6 } & number & 10 & 3728 & 3473 & 4537 \\
\hline & percentage & $0.2 \%$ & $33.3 \%$ & $33.7 \%$ & $70.8 \%$ \\
\hline Cox A10 & number & 11 & 450 & 167 & 457 \\
\hline
\end{tabular}




\begin{tabular}{|llllll|} 
& percentage & $0.2 \%$ & $4.0 \%$ & $1.6 \%$ & $7.1 \%$ \\
\hline \multirow{2}{*}{ Other enteroviruses } & number & 3701 & 3215 & 1676 & 1147 \\
\cline { 2 - 6 } & percentage & $76.2 \%$ & $28.8 \%$ & $16.3 \%$ & $17.9 \%$ \\
\hline
\end{tabular}

*Comparison of HFMD patients with age group, gender and hosting classification in 2017-2020 by chisquare test.

**Significant statistical differences, $\mathrm{P}<0.001$.

$\star \star \star$ No statistically significant differences, $\mathrm{P}>0.05$.

Table 2. The spatial distribution of HFMD annual incidence of districts and counties in Kunming, 20172020.

\begin{tabular}{|lllll|}
\hline Years & 2017 & 2018 & 2019 & 2020 \\
\hline Number & 4855 & 11182 & 10311 & 6406 \\
\hline Incidence & $71.58 / 100000$ & $163.24 / 100000$ & $148.37 / 100000$ & $92.17 / 100000$ \\
\hline Location & & & & \\
\hline Anning & 39.72 & 53.54 & 65.55 & 41.39 \\
\hline Chenggong & 82.48 & 245.82 & 302.61 & 154.94 \\
\hline Dongchuan & 31.10 & 65.49 & 51.93 & 37.54 \\
\hline Fumin & 28.75 & 74.00 & 38.36 & 33.96 \\
\hline Guandu & 176.27 & 384.51 & 344.32 & 204.89 \\
\hline Jinning & 38.51 & 89.59 & 68.95 & 36.86 \\
\hline Luquan & 14.97 & 78.17 & 45.33 & 81.02 \\
\hline Panlong & 67.44 & 149.05 & 133.22 & 77.90 \\
\hline Shilin & 1.52 & 11.37 & 9.02 & 5.26 \\
\hline Songming & 76.92 & 167.99 & 156.58 & 73.39 \\
\hline Wuhua & 75.50 & 154.86 & 125.78 & 102.87 \\
\hline Xishan & 108.87 & 235.78 & 217.55 & 131.79 \\
\hline Xundian & 19.27 & 70.68 & 52.14 & 32.24 \\
\hline Yiliang & 21.37 & 60.74 & 59.76 & 17.01 \\
\hline
\end{tabular}

\section{Figures}




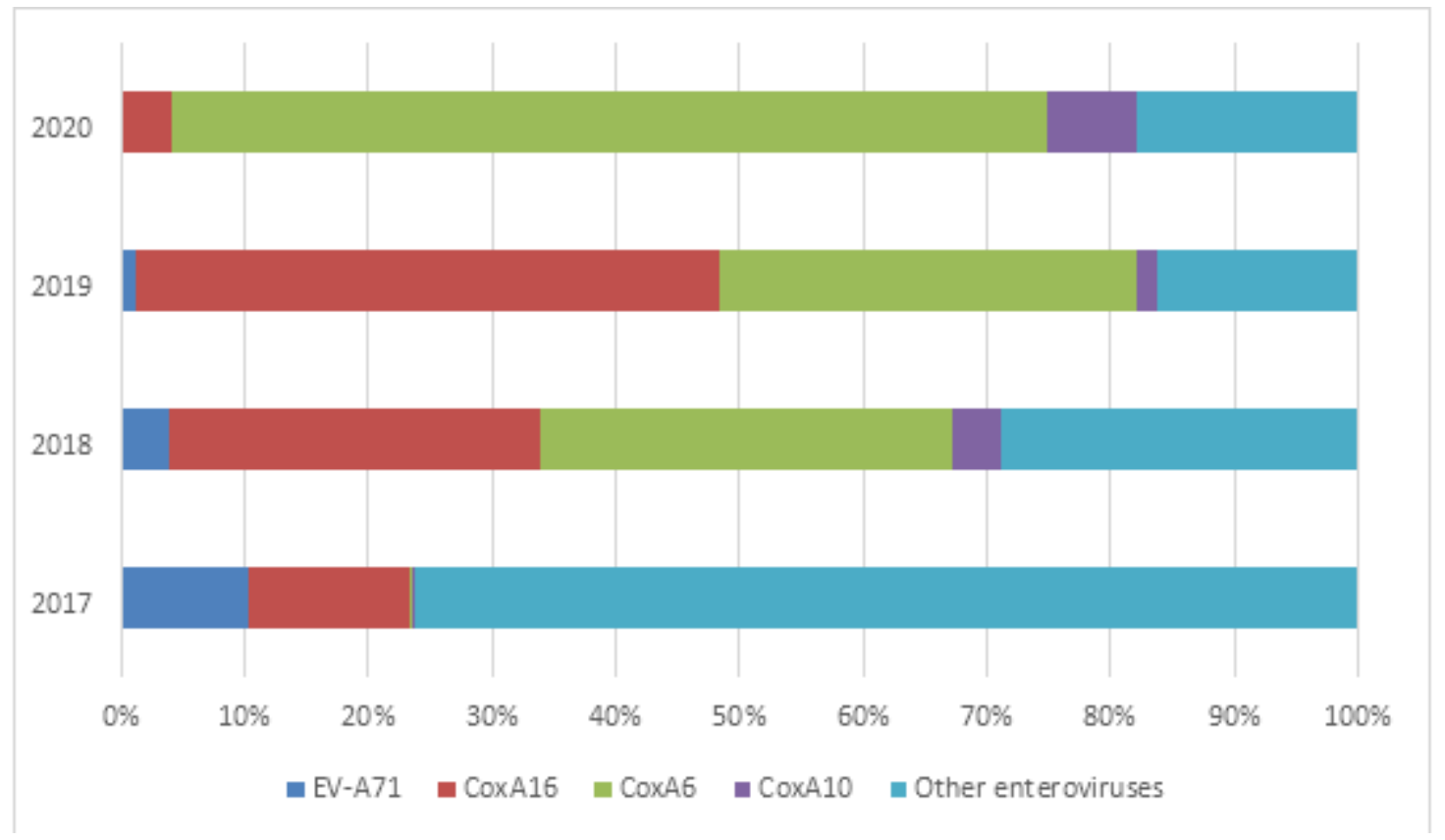

Figure 1

The annual proportion of positive enteroviruses of HFMD in Kunming, 2017-2020.

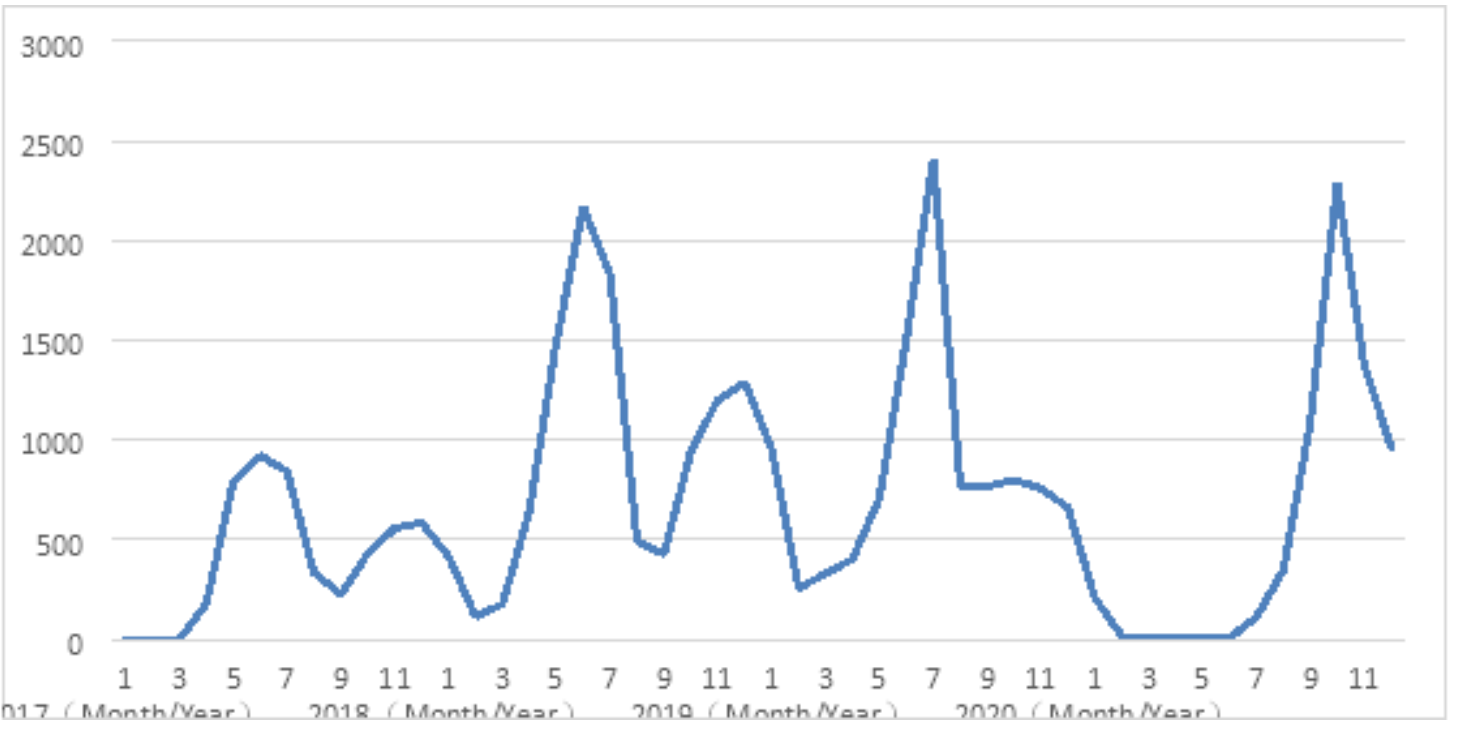

Figure 2

The temporal distribution with periodic peak of HFMD in Kunming, 2017-2020. 


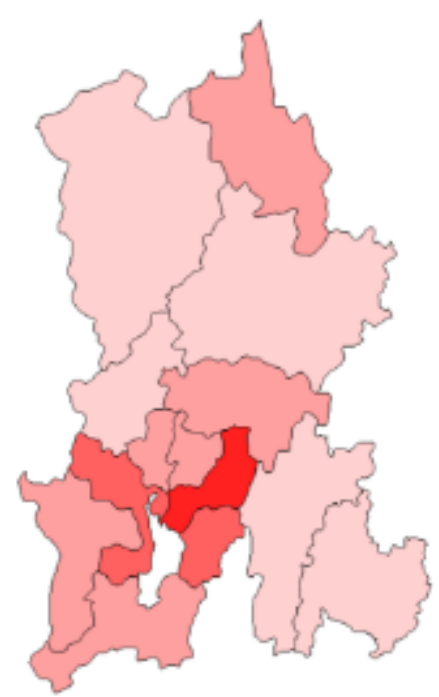

2017

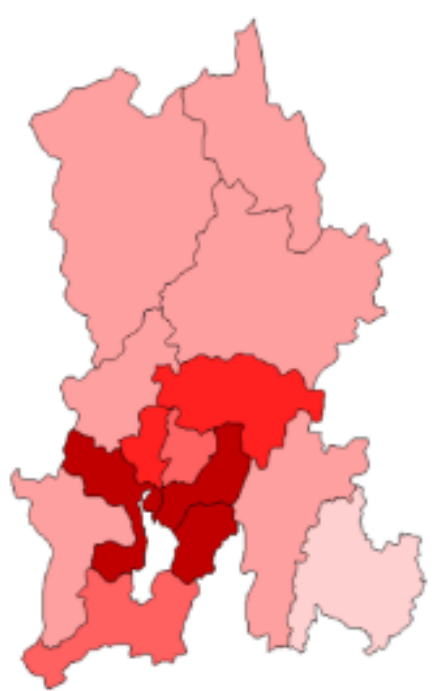

2018

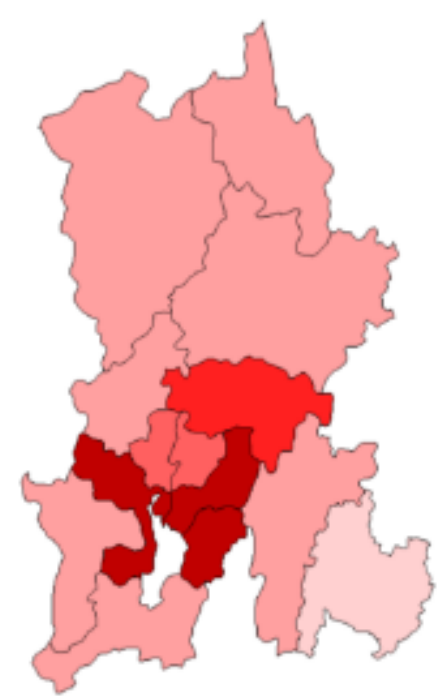

2019

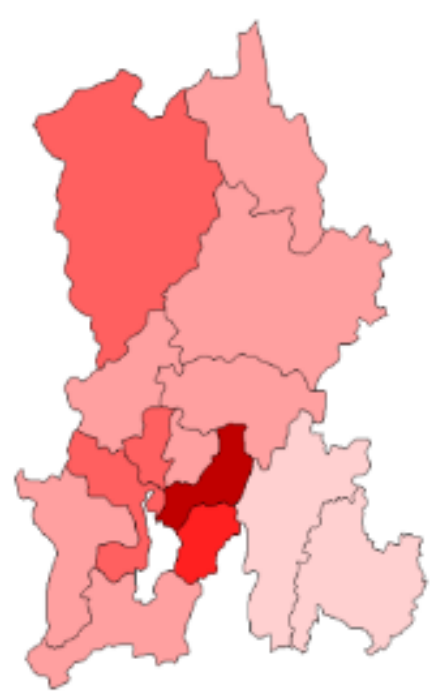

2020

Incidence (/100000)

$\geq 200$

150-200

80-150

30-80

0-30

\section{Figure 3}

The spatial distribution map of HFMD annual incidence of districts and counties in Kunming, 2017-2020. The number of cases of HFMD mainly concentrated in Guandu, Xishan, Wuhua, Panlong and Chenggong, and the high incidence of HFMD areas were Guandu, Chenggong and Xishan.

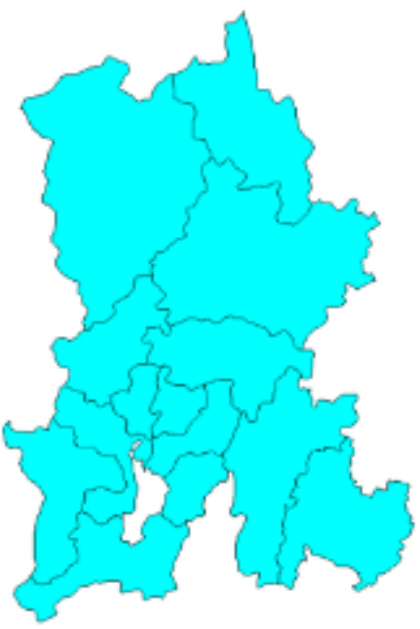

2017

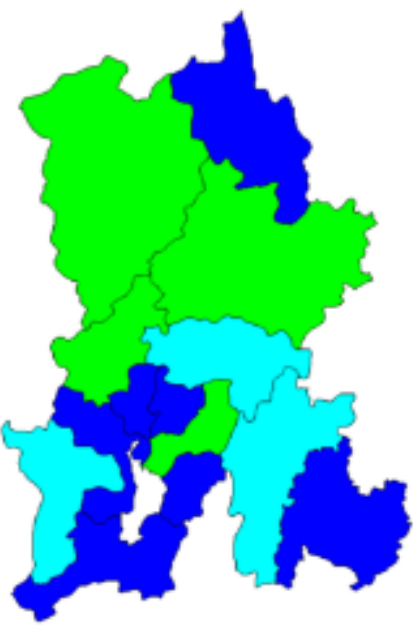

2018

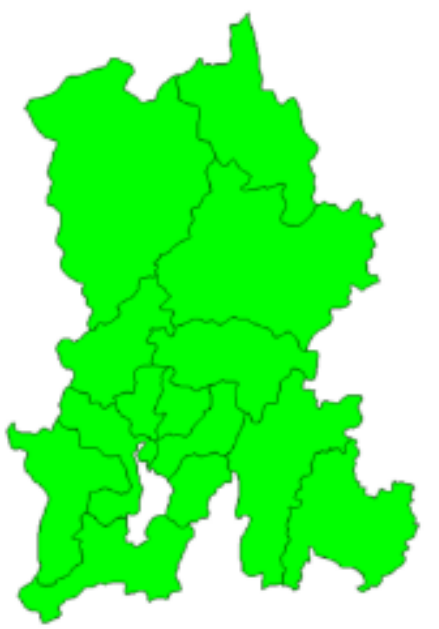

2019

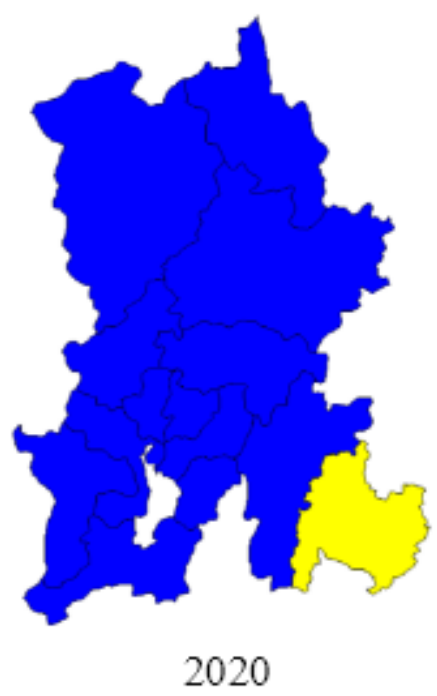

2020

Dominant enteroviruses 


\section{Figure 4}

The spatial distribution map of other enteroviruses as dominant enteroviruses of districts and counties in Kunming, 2017-2020. In 2017, the areas with high proportion of other enteroviruses as dominant enteroviruses were Shilin (100\%), Yiliang (94.7\%), Anning (89.3\%), Chenggong (85.1\%) and Luquan (79.0\%). In 2018, Cox A16 was mainly prevalent in Luquan (43.5\%), Xundian (39.3\%), Fumin (37.6\%) and Guandu (33.2\%); Cox A6 was mainly prevalent in Shilin (60.0\%), Dongchuan (49.5\%), Jinning (45.8\%), Chenggong (37.8\%) and Wuhua (36.5\%). In 2019, the areas with high proportion of CoxA16 as dominant enteroviruses were Jinning (59.9\%), Yiliang (55.8\%), Chenggong (49.5\%), Panlong (48.4\%) and Anning (48.2\%). In 2020, Cox A6 was mainly prevalent in Luquan (77.4\%), Dongchuan (75.7\%), Anning (74.5\%), Fumin (74.1\%) and Xundian (73.4\%); Cox A10 was mainly prevalent in Shilin (50.0\%), Songming (19.1\%) and Yiliang (17.1\%). 